\section{Ophthalmo-Meeting Basel, 20. bis 21 . Oktober 2000}

\section{Funktionsdiagnostik und Neuroophthalmo- logie im Kindes- und Erwachsenenalter}

\section{Huber-Spitzy}

Was als kleine Fortbildungstagung vor ein paar Jahren seinen Anfang genommen hat, ist mittlerweile zu einer großen, sehr informativen Veranstaltung geworden. Das Interesse an diesem Meeting war so groß, dass fast hundert Interessenten abgelehnt werden mussten, um die räumlichen Möglichkeiten nicht zu überfordern und den intimen Charakter zu wahren. Neben Übersichtsvorträgen über Pupillenstörungen und Erkrankungen des Opticus imponierten besonders die Vorträge über bildgebende Verfahren. Rausch (Basel) berichtete über die fMRI (funktionelle Magnetresonanz-Tomographie), mit deren Hilfe der klinische Verlauf von vaskulär bedingten Sehstörungen beobachtet werden kann. Diese Technik ist nicht invasiv und ermöglicht die Unterscheidung zwischen primär durch den Infarkt geschädigten Gebieten und ihrer
Umgebung, die zwar auch mitbetroffen ist, sich jedoch mit der Zeit wieder erholen kann. Zrenner aus Tübingen brachte einen sehr pragmatischen Überblick über die Anwendungsmöglichkeiten und Grenzen der Elektroretinographie, und hob besonders die Bedeutung des multifokalen ERG's bei isolierten Maculaveränderungen hervor. Auch die Vorträge über zentrale Augenbewegungsstörungen, die Untersuchungsmethoden und Hintergründe der visuellen Aufmerksamkeitsstörungen bei Neglect waren sehr praxisnahe und informativ. Zum Schluss berichtete Flammer aus Basel über die Zusammenhänge zwischen Entzündung und Durchblutungsstörung. Chronisch verlaufende Entzündungen führen häufig zu einem sekundären vasospastischen Syndrom, dessen Vorhandensein durch den Anstieg von Endothelin im Plasma bewiesen werden kann. Dies spielt besonders bei Autoimmunkrankheiten wie Multipler Sklerose oder Arteriitis temporalis eine Rolle. Hier könnte gerade bei diesen Erkrankungen ein wichtiger Diagnose-, aber auch Therapieansatz liegen.

Von den meisten Vorträgen war zwar vieles Wiederholung etablierten Wissens, aber gerade dieses Aktivieren des Passivwissens war für den praktizierenden Augenarzt sehr wertvoll. 